\title{
Carte d'assuré: la position de la FMH
}

\section{Judith Wagner*}

Judith Wagner dirige le nouveau service d'état-major «e-Health» du Secrétariat général de la FMH.
En adoptant l'article 42a (voir encadré) de la LAMal, le Parlement a posé les bases de ce qu'il est convenu d'appeler la «carte d'assuré». L'Office fédéral de la santé publique a maintenant élaboré les dispositions d'ordonnance relatives à cet article 42a, lesquelles seront probablement déjà en cours de consultation au moment de la parution du présent article.

Il ressort clairement des procès-verbaux des débats aux Chambres qu'en prévoyant une carte d'assuré, le Parlement a surtout pensé à une simplification des processus administratifs, notamment au niveau de la facturation. Il s'avère également clair que le $4^{\mathrm{e}}$ alinéa vise à préparer l'introduction d'une «carte de santé» et à promouvoir de façon générale le domaine «e-health» (services de santé en ligne) en Suisse. Les principes actuels de la LAMal (critères EAE, tiersgarant, franchises élevées à option, modèles de Managed Care à caractère facultatif) n'ont pas été abordés dans le cadre de ces débats.

La FMH accepte le principe de l'introduction d'une carte d'assuré et soutient l'élaboration de solutions praticables [1]. Elle a également reconnu le potentiel du «e-health» en général et soumettra pour vote à la prochaine Chambre médicale un projet de «carte de professionnel de la santé», carte conçue comme un élément d'une gestion électronique de la santé et comme un élargissement de l'actuelle carte de médecin. La FMH attire toutefois expressément l'attention sur les problèmes liés à l'introduction d'une carte d'assuré et, en particulier, à la gestion de données médicales sur une telle carte. Fondamentalement, quelle que soit la solution retenue, celleci devra impérativement garantir la protection du patient. En outre, une solution judicieuse et vraiment applicable dans la pratique devra être trouvée pour les fonctions administratives de la carte d'assuré et ce, d'un commun accord avec le corps médical. En effet, ce sont les médecins qui, en fin de compte, travailleront avec cette carte.

\section{Carte d'assuré et carte de santé}

Selon le principe envisagé, la carte d'assuré est délivrée par les assureurs à toutes les personnes affiliées à l'assurance-maladie obligatoire. Elle est la propriété de l'assureur concerné et celui-ci pourrait en exiger la restitution après son arrivée à échéance. Un patient pourrait en outre, par exemple, posséder différentes cartes établies par divers assureurs pour les assurances de base et complémentaires. Un changement d'assurance entraînerait l'émission d'une nouvelle carte par le nouvel assureur.

Position de la FMH à ce sujet:

- Une carte d'assuré devrait servir uniquement des objectifs administratifs.

- Le concept d'une carte d'assuré devrait être très clairement séparé de celui d'une carte de santé (ne fût-ce que pour des raisons de protection des données, mais aussi pour des motifs pratiques et de financement).

- Une carte d'assuré (mais aussi une carte de santé) ne devrait contenir aucune donnée médicale, car il n'est pas possible de garantir

\section{Art. 42a Carte d'assuré}

1 Le Conseil fédéral peut décider qu'une carte d'assuré portant un numéro d'identification attribué par la Confédération soit remise à chaque assuré pour la durée de son assujettissement à l'assurance obligatoire des soins. La carte contient le nom de l'assuré et un numéro d'assurance sociale attribué par la Confédération.

2 Cette carte comporte une interface utilisateur; elle est utilisée pour la facturation des prestations selon la présente loi.

3 Le Conseil fédéral règle, après consultation des milieux intéressés, les modalités d'introduction de la carte par les assureurs, ainsi que les standards techniques qui doivent être appliqués.

4 Moyennant le consentement de l'assuré, la carte contient des données personnelles auxquelles peuvent avoir accès les personnes qui y sont autorisées. Le Conseil fédéral définit, après avoir consulté les milieux intéressés, l'étendue des données pouvant être enregistrées sur la carte. II règle l'accès aux données et leur gestion. 
l'actualité et l'exhaustivité de telles données et celles-ci pourraient être perdues si la carte est égarée.

- En raison de leur aspect facultatif et de leur actualité et exhaustivité incertaines, des données médicales figurant sur la carte d'assuré, telles que prévues au $4^{\mathrm{e}}$ alinéa de l'article $42 \mathrm{a}$, n'auraient qu'une utilité extrêmement limitée. Il faudrait également garantir que ces données médicales soient facilement accessibles dans un cas d'urgence, mais qu'elles ne le soient pas du tout en cas de perte ou de restitution de la carte à l'assureur.

- Le droit des patients à l'autodétermination en matière d'information et le principe de proportionnalité (s'agissant de l'enregistrement de données relatives à la personne) doivent absolument être respectés.

La FMH considère le mélange de différents aspects d'une "carte d'assuré» et d'une «carte de santé», tel qu'il est fait à l'art. 42a LAMal, comme extrêmement problématique pour la mise en œuvre de ce projet.

\section{Utilisation de la carte d'assuré: procédure de consultation des données en ligne}

La loi prévoit le recours à la carte d'assuré pour la facturation des prestations selon la LAMal. Dans ce contexte, les discussions portent actuellement sur une procédure qui permettrait d'utiliser la carte pour consulter en ligne chaque semestre, chez l'assureur, les données relatives à l'obligation de prise en charge de ce dernier, de même que les données les plus récentes concernant l'assuré et sa couverture d'assurance. Le logiciel utilisé générerait aussi un numéro qui apparaîtrait sur la facture, de manière analogue aux cartes de crédit. Un objectif possible de cette procédure pourrait être de garantir une transcription directe de données des assureurs sur la facture, et donc d'améliorer la qualité des informations figurant sur celle-ci (ce qui permettrait d'éviter des erreurs de lecture, d'écriture et de transfert et d'assurer des données conformes à la situation actuelle de l'assuré).

Position de la FMH à ce sujet:

- Le fait qu'un patient consulte un médecin fait en soi déjà partie du secret médical, c'est-àdire que l'information sur cette visite ne doit pas automatiquement être transmise à l'assureur.

- Il faut continuer à faire une distinction claire entre la relation patient-assureur et la relation patient-médecin.
- Le patient doit conserver la possibilité de ne décider qu'en fin d'année d'envoyer une facture à l'assureur.

- Il convient de définir les objectifs de la carte d'assuré de manière concertée, claire et vérifiable, puis de trouver des solutions appropriées pour atteindre ces objectifs. Le recours à une carte d'assuré ne doit pas devenir une fin en soi.

La FMH part de l'hypothèse que l'utilisation de la carte d'assuré pour la facturation des prestations devra simplifier les processus et n'occasionner aucune administration supplémentaire. Par conséquent, un tel procédé ne doit générer aucune charge administrative additionnelle et le bénéfice que l'on peut en retirer doit être attesté. Il faut prévoir des solutions simples et applicables aux différentes situations thérapeutiques (p.ex. analyses de laboratoire, pathologie, consiliums, visites à domicile, cas d'urgence, prestations sur mandat, procédure pour médecins agréés, oubli de la carte par le patient, etc.). Il faut aussi mettre à disposition un procédé équivalent permettant de travailler hors ligne (les médecins en cabinet privé ne doivent pas être contraints, entre autres pour des raisons de sécurité, de se connecter à l'internet pour consulter les données nécessaires).

Reste ouverte, par ailleurs, la question de savoir quelles seraient les conséquences d'une situation de non-obligation de rembourser de la part de l'assureur!

\section{Financement}

Une analyse coûts-bénéfices effectuée par Debold \& Lux [2] montre que la carte d'assuré n'apportera des avantages qu'aux assureurs et pas aux cabinets médicaux, lesquels (contrairement aux hôpitaux) n'en auront que les charges. La solution de financement promise par le Conseiller fédéral Couchepin à la table des négociations n'a pas encore été élaborée. La FMH exige toutefois fermement la mise en place d'une telle solution.

\section{Carte de professionnel de santé, cartes d'institutions et autres cartes}

L'utilisation de la carte d'assuré rendra indispensable une carte parallèle chez les prestataires de soins, à savoir des cartes attestant électroniquement que les fournisseurs de prestations sont reconnus comme tels (authentification). Selon leur utilisation, ces cartes devront être munies de fonctionnalités différentes («health professional card» ou «carte de professionnel de santé» pour les médecins, carte d'institution pour le person- 
nel administratif des hôpitaux et des cabinets médicaux ainsi que cartes spécifiques pour le personnel paramédical). La FMH a l'intention de remettre aux médecins une carte de professionnel de santé, en fait une carte électronique de médecin, complétée selon les besoins par des cartes à fonctionnalités partielles. Cette carte sera interopérable avec la carte d'assuré. Les exigences posées doivent être évaluées soigneusement: la FMH met par exemple en question la nécessité d'une signature électronique qualifiée pour les saisies sur la carte d'assuré.

\section{Conclusions}

La FMH relève très clairement les points critiques de l'introduction d'une carte d'assuré et ne manquera pas de les mentionner lors de la procédure de consultation. La prise en compte des problèmes soulevés est évidemment une condition de la participation des médecins à la mise en place du projet.
La gestion électronique des données de santé (e-health) ne pourra être encouragée et propagée dans toute la Suisse que si l'on applique la loi judicieusement et utilement. Toute autre démarche serait contre-productive.

Finalement, «un projet tient et ou ne tient pas en fonction de son taux d'acceptation par les utilisateurs» [3]: cette remarque pleine de bon sens devrait aussi être valable pour la Suisse!

\section{Références}

1 de Haller J. Carte d'assuré: que pense, que fait la FMH? Bull Méd Suisses. 2006;87(33):1399.

2 Debold \& Lux Beratungsgesellschaft für Informationssysteme und Organisation im Gesundheitswesen mbH. Die Versichertenkarte und der Aufbau einer Telematikinfrastruktur, Kosten-NutzenAnalyse. www.bag.admin.ch/themen/kranken versicherung/00305/00306/index.html?lang=de.

3 Bundesärztekammer. Deutschland: Diskussion um elektronische Gesundheitskarte. Schweiz Ärztezeitung. 2006;87(35):1513. 